\title{
Doctrine des signatures et technologies graphiques au seuil de la modernité
}

Francesco Panese

\section{Summary}

The "Doctrine of signatures" occupies an important position in the scientific and medical thinking from the 16 th century onwards. In a universe conceived as a vast system of correspondences, in particular between our world and God's will, the signatures are the visible marks of the purpose of divine creation. In therapeutic practices they indicate the virtues of plants by their resemblance to specific organs they are supposed to be able to cure. This Doctrine, like the medical practices it served, is often relegated to a pre-modern knowledge. Shifting the perspective from its doctrinal content to its practical applications in medical treatises, this article suggests that the graphical and scriptural treatment of signatures by Oswald Crollius (ca 1560-1609), Giovanni Battista Della Porta (1534-1615) and Wolfgang Ambrosius Fabricius (1653) allowed the invention of an instrument which, by formalising and systematising the correspondences between plants and organs, made possible a practical use in which metaphysical and religious justifications were not explicitly involved any more, precisely these justifications which resulted in confining this doctrine outside of modernity.

\section{Résumé}

La doctrine des signatures occupe une position importante dans les sciences et la médecine dès le $\mathrm{XVI}^{\mathrm{e}}$ siècle. Dans un univers conçu comme un vaste système de correspondances, notamment entre le monde sensible d'ici-bas et la volonté du Créateur, les signatures des choses sont les signes visibles de

Francesco Panese, Université de Lausanne, SSP-ISCM, BFSH 2, CH-1015 Lausanne (Francesco.Panese@iscm.unil.ch). 
leur appartenance au plan divin. Dans le domaine thérapeutique, elles indiquent les vertus des plantes en «exhibant» leur ressemblance avec les organes qu'elles sont susceptibles de soigner. Cette doctrine, comme les pratiques médicales qui l'accompagnent, est souvent reléguée à une époque du savoir d'avant la «modernité». En déplaçant le point de vue du contenu «doctrinal» à sa mise en pratique dans des traités destinés à l'usage des médecins, cet article suggère que le traitement graphique et scripturaire des signatures chez Oswald Crollius (c. 1560-1609), Giovanni Battista Della Porta (15341615) et Wolfgang Ambrosius Fabricius (1653) a produit un instrument qui formalise et systématise les correspondances entre plantes et organes, et a permis un usage pratique de la doctrine qui puisse faire l'économie des justifications métaphysiques et religieuses, celles-là même qui ont conduit à cantonner cette doctrine hors de la «modernité».

\section{Introduction}

$\mathrm{Au} \mathrm{XVI}{ }^{\mathrm{e}}$ siècle, le médecin suisse Paracelse propose une interprétation du monde et une conception de la connaissance profondément inscrites dans son époque, et qui féconderont le monde savant, surtout médical, durant au moins un siècle, avant d'être marginalisées par l'affirmation du regard scientifique moderne. Selon lui la connaissance de la nature consiste, in fine, à renouer les fils de la Création divine, notamment à partir de l'apparence sensible des choses:

L'ordre de la nature veut que les signes extérieurs manifestent l'œuvre interne et les vertus. Il en a plu ainsi à Dieu, afin que rien ne demeure caché, et que ce qui se trouve celé dans toutes les créatures soit révélé par les sciences. On pourrait s'étonner et se demander pourquoi Dieu a institué que, par l'art, l'homme apprenne à connaître ce qui se trouve ainsi occulté. La raison en est que le corps de l'homme est animé et que l'homme doit apprendre à connaître l'élément moteur que Dieu y a déposé. Dieu de la sorte n'a pas fait que la science demeure vaine, mais au contraire, a voulu que la connaissance de toutes les vertus et de tous les secrets passe par elle; c'est donc par elle que nous apprenons à connaître l'essence invisible des choses. ${ }^{1}$

Cette conception du monde et les pratiques de connaissance qui lui sont associées construisent le monde sensible comme un lieu où les choses portent les marques visibles du Créateur, les signatures de leurs appartenances multiples et réciproques au plan divin. Cette nouvelle économie du visible occupe une position charnière dans l'histoire de l'émergence des sciences modernes. D'un côté, la connaissance reste inséparable d'une théologie qui prend le chemin de la divinatio pour comprendre et décrire le monde comme

1 Astronomia Magna, trad. et cit. dans: Braun 1994. 
un vaste système de correspondances entre des éléments et des registres hétérogènes. Du côté de ses pratiques concrètes, lorsque l'analogie entre le monde sensible et le plan divin s'inscrit de manière figurative au cœur des fruits de la Création, l'étude des signatures est une connaissance basée sur la description du visible et participe à l'affirmation du sens de la vision comme sens privilégié de la connaissance. L'histoire des technologies de l'intelligence semble alors devancer celle des idées ${ }^{2}$. La modernité scientifique semble ainsi s'esquisser dès le $\mathrm{XVI}^{\mathrm{e}}$ siècle dans le travail de savants qui, à sa suite, entreprennent de déchiffrer les physionomies de l'homme, des plantes ou des animaux comme les signes des Ecritures transposées en figures dans la Nature.

\section{Une conception analogique du monde}

Il est utile de rappeler ici les figures essentielles à travers lesquelles le savoir de la ressemblance se déploie, et que Foucault a décrites dans des pages lumineuses: conventio, aemulatio, analogie, sympathie et antipathie se relaient pour décrire un monde où «la nature et le verbe peuvent s'entrecroiser à l'infini, formant pour qui sait lire comme un grand texte unique», la «prose du monde» ${ }^{3}$. Ces figures expriment chacune la trame serrée du jeu des correspondances à travers des êtres singuliers. La conventio désigne la parenté par le voisinage, une relation qui lie de proche en proche des entités hétérogènes. La forme d'un œil qui se dessine visiblement sur une plante peut alors désigner une correspondance entre l'organe de la vue et les vertus thérapeutiques du végétal. Mais la correspondance peut aussi découler d'un «rapport d'émulation [par lequel] les choses peuvent s'imiter d'un bout à l'autre de l'univers sans enchaînement ni proximité: par sa réduplication en miroir, le monde abolit la distance qui lui est propre; il triomphe par là du

2 Nous reprenons la notion de «technologie» à S. Shapin et S. Schaffer, qui ont décrit dans leur ouvrage célèbre Léviathan et la pompe à air: Hobbes et Boyle entre science et politique l'émergence de l'expérimentalisme anglais sous l'angle de la mise en place de trois technologies permettant d'articuler les observations de la nature, leur communication et leur discussion: une technologie matérielle qui correspond à la fabrication et à la mise en œuvre de l'instrumentation scientifique; une technologie littéraire qui implique des formes spécifiques de communication verbale et visuelle des faits à ceux qui n'ont pu en être les témoins directs; une technologie sociale qui définit les conventions que les philosophes de la nature doivent respecter dans leurs relations mutuelles afin de pouvoir confronter de manière valable leurs revendications de connaissance. Voir Shapin/Shaffer 1993, chap. 2 en particulier. En ce qui concerne l'importance des technologies graphiques sur le plan des développements intellectuels, nous renvoyons aux travaux désormais classiques de Goody 1979 et d'Eisenstein 1979.

3 Foucault 1966 (chap. 2: «La prose du monde», 49). 


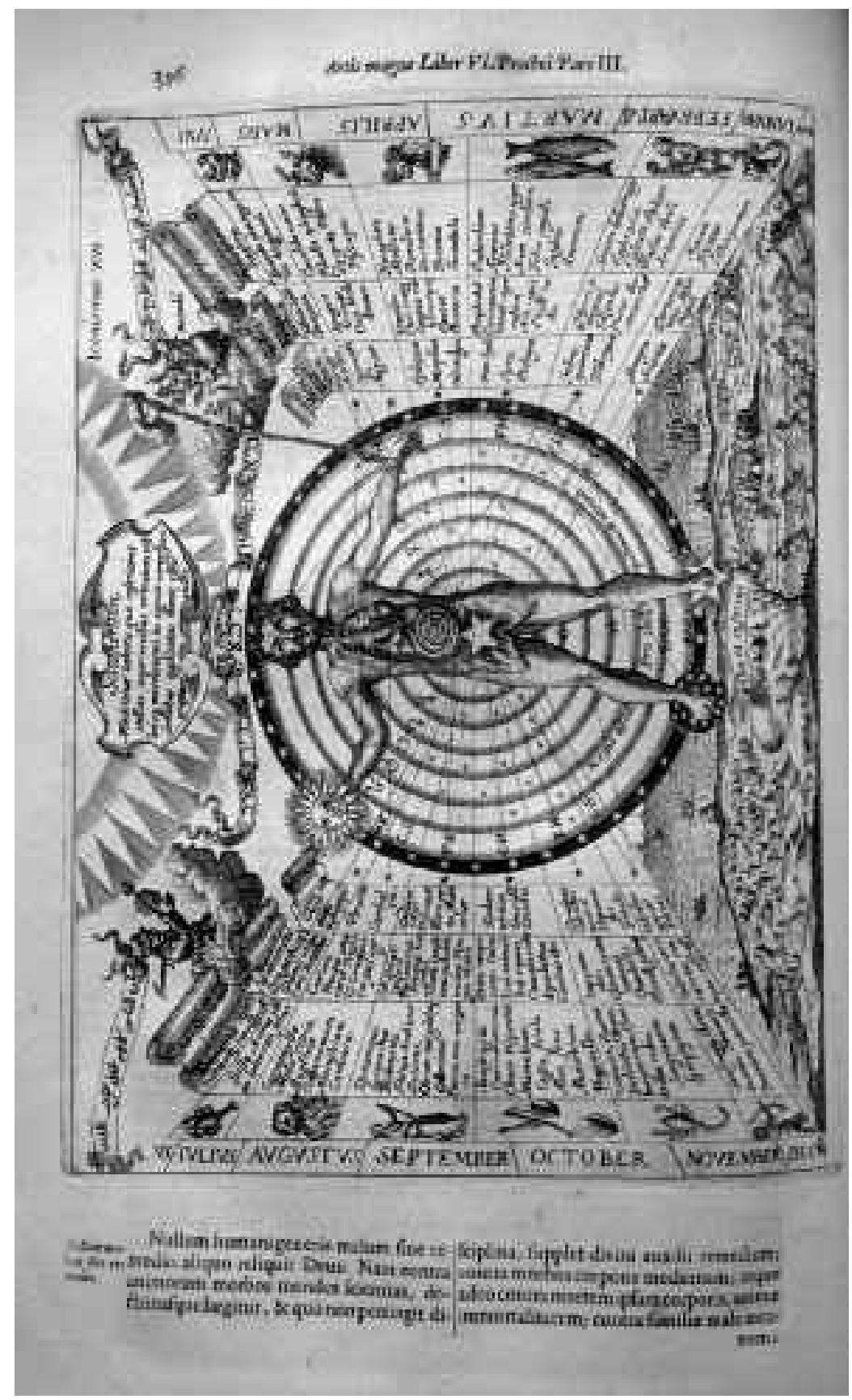

Fig. 1. Athanasius Kircher, Ars magna lucis et umbrae (1671) 396: Sciatericon. 
lieu qui est donné à chaque chose» ${ }^{4}$. Ce sont ces rapports d'aemulatio qui tissent le réseau des correspondances, par exemple entre les étoiles et la vie des hommes ou des végétaux et, partant, entre les organes humains et les vertus curatives des plantes, comme l'illustre ce passage d'Oswald Crollius (c. 1560-1609):

[...] les estoiles sont la forme \& la matrice de toutes les herbes, \& chasque estoile du ciel, n'est autre chose que la confuse et spirituelle préfiguration d'une herbe, telle qu'elle la représente, \& tout ainsi que chaque herbe ou plante est une estoile terrestre regardant le ciel, de mesme aussi chaque estoile est une plante céleste en forme spirituelle, laquelle n'est différente des terrestres que par la seule matière, à raison dequoy tous les estoiles predisent les maladies futures par leurs excréments, \& nostoch, \& aussi les plantes et herbes célestes sont tournées du costé de la terre \& regardent directement les herbes qu'elles ont procreées, leur influant quelque vertu particulière, à cause de la sympathie mutuelle. ${ }^{5}$

La sympathie et son contraire constituent pour ainsi dire le moteur de la similitude, le phénomène en vertu duquel un être peut reproduire les modifications d'un autre être par influence ou par imitation. C'est la sympathie et l'antipathie qui rapprochent et éloignent les choses tant sur le plan de l'empirie que sur celui de la pensée. La parenté entre les êtres ne relève pas forcément des ressemblances évidentes qui s'offrent d'emblée au regard. Les liens que toutes les choses créées entretiennent entre elles se nouent sur un espace plus abstrait de rapports, qui forme la condition de leurs manifestations visibles, empiriques, saisissables par le spectateur du monde. La raison qui structure cet espace de correspondance est l'analogie. Elle est le pôle heuristique de la position épistémique de la similitude. La longue tradition de l'homme-microcosme illustre bien l'exercice d'une telle pensée, en ramenant analogiquement les êtres animés et inanimés à la matrice humaine. Le corps humain devient alors le centre d'un réseau de signification qui peut s'appliquer de proche en proche à l'univers tout entier. Cette conception est bien illustrée par une représentation tardive d'Athanasius Kircher (1601-1680) qui installe l'Homme organique au centre de ce «cadran sciatérique», comme la référence analogique de toute chose (fig. 1$)^{6}$. De ses parties partent les liens qui les associent successivement aux planètes, aux étoiles, aux plantes capables de les soigner, aux signes astrologiques et au rythme du temps ${ }^{7}$.

4 Foucault 1966, 34sq.

5 Crollius 1976.

6 Kircher 1671.

7 Comme, selon cette conception, l'homme est le représentant suprême de la Création, c'est aussi un être panzoïque (pan zôon) qui possède à la perfection toutes les propriétés et aptitudes animales. Les traités de physiognomonie sont le plus souvent construits selon ce système analogique, comme le fameux De humana physiognomia de Della Porta (1586), qui fait de l'homme le siège de la diversité animale et, en retour, l'énigme que cette diversité permet de déchiffrer. 
La conception analogique du monde est sans doute un des traits caractéristiques des ouvrages savants du $\mathrm{XVI}^{\mathrm{e}}$ siècle, des écrits de Paracelse (1493-1541) aux traités zoologiques de Pierre Belon (1517-1564). Chacun à sa manière vise à produire un ordre du monde qui, au contraire de l'ordre classique, ne consiste pas à déployer en tableau les réseaux d'identités et de différences entre les choses, mais à scruter le même dans l'autre afin de recomposer la longue chaîne des créatures engendrées par l'intelligence divine. Toute étude particulière s'inscrit dans ce projet à visée globale. Comme le dit Céard dans son commentaire de l'Histoire de la nature des oyseaux de Belon (1555), «malgré sa discrétion et son souci de ne traiter que des oiseaux, [Belon] tient cette étude pour une partie seulement d'un examen plus vaste de l'harmonie intime des choses; il s'agit, à travers l'intelligence de la diversité, de saisir l'unité profonde qui la maintient et qui s'exprime en elle» ${ }^{8}$.

\section{Une quête inachevée}

Pour ces naturalistes, l'analogie est l'outil d'interprétation d'un monde qui se donne à voir de manière énigmatique et partielle. La nature, comme expression sensible de l'ordre de la Création, est envisagée, à l'instar des Ecritures, comme le fruit d'un langage divin qu'il s'agit de déchiffrer. Toute connaissance de «notre machine visible» est alors une exégèse. Les outils de sa compréhension reposent sur l'idée que la trame invisible de la Création ne se manifeste à l'homme que sous la forme de traces: les signatures sont les signes par excellence de ce savoir indiciaire. Elles sont les marques visibles des mouvements cachés qui engendrent les ressemblances entre les choses. La signature scelle la ressemblance. Ce savoir naît ainsi de la rencontre entre deux projets d'écriture: celui du Créateur tout d'abord, qui a imprimé sa marque universelle au cœur des choses particulières; celui des hommes ensuite, qui recueillent ces marques pour assigner aux choses les places qui leur reviennent dans l'ordre supérieur de la Création, l'horizon ultime de leur travail d'interprétation. Le plan divin est ainsi l'asymptote des longues chaînes de ressemblances qui relient toutes les choses créées, mais qui restent impuissantes, sur le plan du savoir, à saisir la totalité. Selon une image récurrente à l'époque, le savant comme le médecin doivent, à leur manière, remonter pas à pas l'échelle de Jacob:

8 Céard 1980. 
[...] quiconque des médecins n'a ce fondement, \& philosophique Alphabeth, ne mérite de porter ce beau nom: ces charactères \& signatures naturelles, lesquelles nous avons dès notre création, non marquées avec l'ancre, ains avec le doigt de Dieu (chasque creature estant un livre de Dieu) sont la meilleure partie, par laquelle les choses occultes sont rendues visibles et descouvertes. [...] Il est asseuré que l'on compte trois mondes, \& que ces trois ne sont qu'un universel, parce qu'ils sont l'un dans l'autre, sçavoir Dieu, les Anges, \& nostre machine visible, l'inferieur est gouverné par le superieur, duquel il prend l'influxion de ses vertus: tellement que l'archetype mesme \& supreme fabricateur nous influë les vertus de sa toutepuissance, par les anges, Cieux, estoiles, elements, animaux plantes, \& pierres, au ministere desquelles il a fait et creé ce tout. Mais venons à nostre entrée ou montée, laquelle se fait lors que par eschelle de Jacob nous nous eslevons de bas en haut, c'est-à-dire des choses sensibles aux intellectuelles; des créatures au Createur, montant toujours. ${ }^{9}$

Celui qui n'emprunte pas ce chemin, c'est-à-dire «celuy qui sépare du Créateur la cognoissance des choses créées, n'a seulement que l'ombre des choses créées» ${ }^{10}$. Pourtant, la réponse analogique à l'interrogation sur la marche $\mathrm{du}$ monde est toujours provisoire, voire allusive. Pour les humains, cette remontée a les allures d'un labyrinthe dont le plan ne peut jamais être achevé. Les méandres de la connaissance sont le pôle opposé de l'achèvement de la Création, de son ordre, de sa symétrie, de sa perfection qui se dérobe souvent aux regards myopes des hommes. Le philosophe ou le médecin peuvent bien sûr aiguiser leur vue, mais jamais elle ne pourra pénétrer «sous le voile du silence de la nature», selon la belle formule de Crollius. Dans cette quête, un écart persiste toujours entre le monde et la manière dont il se rend saisissable par les marques que le Dieu y a déposées. C'est dans cet écart que se superposent une science des signes et une discipline de l'interprétation ${ }^{11}$.

Cet épisode de l'histoire humaine de la nature sera bouleversé, on le sait, par l'émergence des sciences modernes, mais ce bouleversement n'a sans doute pas eu les allures d'une rupture rapide et radicale entre deux époques du savoir, deux «épistémès», comme a pu le suggérer Foucault:

[L'épistémè du XVI ${ }^{\mathrm{e}}$ siècle] emporte avec soi un certain nombre de conséquences. Et d'abord le caractère pléthorique et absolument pauvre de ce savoir. Pléthorique puisqu'il est illimité. La ressemblance ne reste jamais stable en elle-même; elle n'est fixée que si elle ne renvoie à une autre similitude, qui en appelle à son tour une nouvelle; de sorte que chaque ressem-

9 Crollius 1976,27. Crollius est très inspiré par la Cabale. Sa quête y ressemble, à la différence importante près que le lieu de la lisibilité de la Création migre des Ecritures aux créatures qui deviennent autant de livres ouverts que seul le médecin véritable pourra lire. Après avoir énoncé le principe de correspondance entre les choses «corporelles» et «spirituelles», en particulier entre le «Soleil» comme «tabernacle» de Dieu, le «Soleil celeste» et le «Soleil terrestre», Crollius conclut: «l'esprit de vie de celuy là [le soleil terrestre] s'appelle esprit caché: mais celuy-ci [le soleil céleste] s'appelle proprement et genuinement en toute chose soulphre, du moins si nous voulons adiouster foy aux doctes Cabalistes, l'estude desquels a esté de monter du signe au signifié; des créatures aux créateur, des Anges à Dieu, \& là se joindre estroitement avec lui, affin que par ce moyen (selon Pythagore) ils se peussent deifier» (Crollius 1976, 35).

10 Crollius 1976, 27.

11 Sur ce point, cf. également Foucault 1966, 44sq. 
blance ne vaut que par l'accumulation de toutes les autres, et que le monde entier doive être parcouru pour que la plus mince des analogies soit justifiée, et apparaisse enfin comme certaine. $^{12}$

Si cette analyse reste convaincante du point de vue de l'interprétation épistémologique de la doctrine des signatures ${ }^{13}$, elle rend mal compte de sa mise en pratique dans des instruments qui servaient de pharmacopée aux médecins. En effet, nous tenterons de montrer que la mise en tableaux systématiques des signatures, associée à la multiplication des figures publiées en grand nombre dès le XVI ${ }^{\mathrm{e}}$ siècle, participe d'une technologie graphique qui, contrastant en cela avec ses fondements doctrinaires, va contribuer à limiter le nombre des associations entre les plantes et les organes pertinentes pour le médecin, à stabiliser textuellement ou figurativement les ressemblances et, de manière plus générale, à domestiquer la «doctrine des signatures» dans un outil de prescription médicale.

\section{Signatures, expérience et connaissance visuelle}

L'épistémè de la similitude, dont la signature fonde la sémiologie, ne renvoie pas, entre la fin du XVI ${ }^{\mathrm{e}}$ et le début du XVII ${ }^{\mathrm{e}}$ siècle, à un supposé savoir herboristique et médical «populaire», bien au contraire. En permettant de donner une évidence visible aux vertus invisibles, la doctrine des signatures trace une ligne de démarcation entre un savoir médical basé sur la vision et l'expérience et un savoir qui relègue au second plan l'observation de la nature au profit des interprétations qui en ont été faites dans la longue tradition des commentaires des textes classiques ou bibliques. ${ }^{14}$ Ceux qui cultivent la doctrine des signatures interviennent alors de manière stratégique et critique dans la reconfiguration du savoir médical de l'époque. C'est le cas du médecin Crollius, qui tente de systématiser cette doctrine afin, entre autres, de réformer le savoir médical de son temps et de «discipliner» ses acteurs. L'ouverture de son Traicté des signatures est de ce point de vue très explicite:

La mienne volonté que les botanistes de nostre temps, lesquels ignorans la forme interne des herbes, n'en recognoissent que la substance materielle, employassent aussi bien leur estude à la cognoissance de leurs signatures, qu'ils sont pour l'ordinaire à la dénomination d'icelles;

12 Foucault 1966, 45.

13 Notons sur ce point que Foucault, lecteur attentif et passionné de Paracelse, de Della Porta et de Crollius, ne commente à aucun moment la seconde partie de Traicté de Crollius ni les figures de Della Porta, sur lesquelles nous allons précisément nous arrêter dans la suite de notre propos.

14 L'affirmation de ce savoir nouveau s'est d'ailleurs également opérée en y important une part du savoir de «l'ordinaire» dont la prise en compte est récurrente dans le travail des nouveaux savants, mais aussi des ingénieurs. Sur ce point, voir Rossi 1996. 
sur quoi ils fondent une infinité de vaines disputes, lesquelles ne sçauroient apporter aucun proffit à la république de médecine. [...] Outre ce ie m'estonne grandement, qu'ils passent sous silence la preuve qui se doit faire par l'industrie du feu, \& couteau anatomique: car le nombre des vertus, qu'ils attribuent à chasques herbes (prinses aux escrits de quelque autre, sans qu'ils en ayent aucune preuve) sont pour la plus grande part fausses, erronées, \& sans aucun fondement: car il n'y a que l'expérience maitresse de toutes choses, laquelle puisse donner un témoignage assez suffisant pour satisfaire à l'entente des médecins, et au contentement des malades. ${ }^{15}$

Dans la ligne de Paracelse, Crollius cherche à fonder un savoir médical en raison et en religion, deux dimensions inséparables sur lesquelles doit se construire à l'époque une connaissance certaine. La doctrine des signatures forme la clé de voûte qui soutient mutuellement la cosmogonie chrétienne et une sémiologie naturelle qui puisse devenir l'outil privilégié de la «république de Médecine». Elle doit permettre selon lui de différencier les pseudomédecins des «médecins véritables», en constituant un savoir à partir de données sensibles et surtout visibles:

Comme l'homme est cognu par ses fruicts, de mesme aussi les plantes sont cogneües par leur signature. Homere appelle les medecins peri pànton ùpeirokhos allòn, d'autant qu'ils doivent tout voir. L'anatomie \& forme des herbes se doit accorder \& correspondre à l'anatomie, \& forme des maladies: car si la physionomie et Chyromancie tant des maladies, que des remedes ne sont essentiellement cogneuës des medecins, à peine feront-ils jamais rien qui vaille, d'autant que la signature est un grand fondement tant pour la medecine que pour la philosophie. ${ }^{16}$

Le règne végétal prend ici la forme d'un système de signes visuels, un espace de vertus figurées à l'intérieur duquel le médecin peut orienter sa pratique thérapeutique. Cette affirmation du sens de la vue est symptomatique de la critique de la médecine aristotélico-galénique menée dès Paracelse. Il en est des plantes curatives comme des malades. Pour qu'une connaissance puisse être considérée comme vraiment fondée, son objet doit être pleinement accessible à la vue du médecin - et au toucher lorsque le mal se dérobe au regard - dans toutes ses caractéristiques sensibles. L'observation visuelle

15 Crollius 1976, 7sq. Plus loin, Crollius en arrive à faire le procès de ceux qui cherchent plus leur propre profit que la guérison des malades: «L'on sçait bien que l'avidité du lucre est telle, qu'elle donne des bonnes inventions pour les sophistiquer \& diversifier en mille façons [les médicaments]; stupides que nous sommes, nous ne tenons compte de l'abondance que Dieu nous en donne en Europe, trop bastante pour subvenir à nos infirmitez, \& d'où cela? si ce n'est qu'on ne veut pas mettre la peine \& diligence qui est requise en tel cas, d'autant que la gravité de nos medecins est venuë en tel point, qu'ils méprisent aussi bien la noirceur du charbon, que la soüille de l'argile. Je laisse à part les Apothicaires, desquels la plus grande partie poussée par la gloire ou avarice, cherche plustost l'escoulement de la bourse du malade, que non pas la restitution de sa santé, d'où arrive (au grand dommage de la république de Médecine, \& au grand peril de la vie des personnes) qu'il n'y a rien de plus cher que ce qui vient delà la mer rouge, ou du fond des Gades, \& des Indes, ou que ce qu'on nous donne à croire en estre venu: ceux qui ont achepté leur mort par quelque grande somme de deniers en pourroient donner un asseuré tesmoignages (s'il leur estait permis d'en revenir dire leur advis)» (Crollius 1976, 19).

16 Crollius 1976, 34. 
constitue ici un instrument de connaissance au service d'une nouvelle forme de certitude. L'espace d'interprétation du médecin - comme celui des nouveaux philosophes de la nature qui se multiplient tout au long du XVII ${ }^{\mathrm{e}}$ siècle - se réduit sensiblement pour devenir un espace idéal de pure visibilité. Dans le trop grand désordre des «langues» qui prétendent dire la nature, il s'agit de retrouver celle que la nature elle-même compose:

[...] Supposons que les plantes puisent parler d'elles mêsme, \& dire les admirables et secrettes vertus, desquelles la nature les a enrichies, asseurement elles ne seront pas entendües de tous, ny leurs facultés si bien manifestées par les escrits cogneus par tout le monde; ou il eust fallu necessairement que les plantes fussent esté toute une nation, ou bien qu'elles eussent parlé en toute sorte de langues: c'est donc assez que la sage nature manifeste subtilement son pouvoir par quelques sympathie \& signatures congneües de tout le monde. N'est-il pas vray que toutes les herbes, plantes, arbres \& autres provenans des entrailles de la terre, sont autant de livres, \& signes magiques, communiquées par l'infinie misericorde de Dieu? ${ }^{17}$

Le travail des naturalistes consiste alors à traduire au mieux ces livres divins. Le faire sans trahir est impossible, mais on peut s'y essayer en poursuivant deux voies complémentaires qui garantissent une plus grande fidélité de la connaissance: l'expérience et l'observation, «l'industrie du feu, \& couteau anatomique». Dans les termes de l'époque, elles sont liées dans la démarche alchimique, qui consiste à séparer et à recomposer des substances naturelles par l'artifice, par l'action de la chaleur notamment ${ }^{18}$. Selon une conception que l'on retrouve chez Francis Bacon, l'expérience alchimique est considérée ici comme le prolongement du sens de la vision qui, comme le feu, décompose et offre une nouvelle visibilité aux choses. Elle constitue un moyen pour dépasser l'observation du monde tel qu'il s'offre immédiatement aux sens et parvenir aux contenus invisibles qui génèrent les formes visibles. Tel est également le rôle des signatures, dont l'observation et la systématisation constituent d'emblée une forme d'expérience.

17 Crollius 1976, 11.

18 Il faut se souvenir ici que la plus grande partie des attaques de l'expérimentalisme contre l'alchimie concernait plus la manie du secret qui la caractérisait que sa démarche expérimentale qui, dans beaucoup de cas, lui était reconnue par les nouveaux philosophes de la nature. Sur ce point, $c f$. Shapin/Shaffer 1993. La conclusion de Paracelse à sa Parenthèse sur les cinq entités résonne de ce point de vue en des termes presque baconiens: «Si vous voulez passer tout à fait pour de bons et habiles médecins, appliquez vous à ne pas perdre votre cause par le style chrétien et païen que vous étudiez, et ne souffrez pas d'être contredits par les médecins ignorants qui s'habillent de rouge et de noir. Car ce sont des Phantastes qui disent des sornettes (nugantur), suivant leur fantaisie, et auxquels nul ne peut se fier. Et, dans cette conclusion, notez qu'il y a deux parties dont l'homme se sert: L'Art et la Fantaisie. L'Art (c'est-à-dire toute raison, sapience et intelligence), procède dans la vérité, qui s'appuie sur la base de l'expérience. Ceux qui s'adonnent aux Phantasmes manquent de base. Car l'opinion préconçue n'est qu'une ambition avouée et manifeste, que vous n'êtes pas sans connaître dans votre entourage. A l'égard de ces deux parties, il convient à l'homme sapient d'être parfaitement accompli et instruit, c'est-à-dire qu'il doit être un homme habile dans l'art, et non un charlatan vêtu de rouge» (Paracelse 1984, 128). 
Le passage entre visibilité indicielle et invisibilité essentielle que permet la signature est bien assuré par la concordance réciproque de l'extérieur et de l'intérieur des choses engendrée par la médiation divine. Pourtant, cette concordance ne relève pas d'une influence «magique» des choses entre elles. La signature relève d'une conception que l'on pourrait dire isomorphique du monde selon laquelle toutes choses ayant été engendrées par un unique créateur, toutes ont été créées selon un même dessein et doivent donc suivre des chemins analogiques ${ }^{19}$. Selon une belle métaphore de Paracelse, la signature peut être comparée au plan d'une maison établi par un charpentier. Cette maison est d'abord le fruit de son imagination et, une fois construite, elle correspondra à sa préfiguration idéale de manière à ce qu'à travers sa forme matérialisée, on pourra remonter à la pensée du charpentier:

Voici quelques exemples qui feront mieux comprendre la science des signatures. Un charpentier qui veut construire une maison se représente d'abord la maison. La maison se fera d'après cette représentation. La forme de la maison manifestera la pensée du charpentier. Ainsi donc personne ne connaît ce qui est dans l'esprit, ou dans la pensée, ou dans la nature, avant que cela n'ait pris forme et figure. Celui qui a l'intention de faire quelque chose le rend manifeste par l'œuvre ou le fruit; ainsi rien ne demeurera caché; tout se révèle de soi-même. Un autre exemple. On façonne une chose d'après la fin qu'elle doit remplir. Si quelqu'un veut construire une tour ou une forteresse, il leur donne la forme qui est la plus appropriée à tenir tête aux ennemis; et quiconque est expert dans l'art des fortifications voit, d'après sa forme, ce que vaut la tour et à quel genre de fortifications elle appartient. ${ }^{20}$

La conception du savoir qui sous-tend la doctrine des signatures est donc une quête de visibilité de la nature traduite en texte lisible par la médiation du travail et de l'expérience de l'«homme sapient». Fidèle à Paracelse, Crollius inscrit la doctrine des signatures - et par là tout son projet de sémiologie médicale - dans la filiation d'autres mantiques basées sur l'interprétation visuelle:

Paracelse, pere des secrets (nom qu'il a mérité entre tous les medecins) exhorte de tout son pouvoir ceux lesquels veulent acquerir la vraye \& parfaicte science de la médecine, qu'ils employent toute leur estude à la cognoissance des signatures, hieroglyphes et charactères; outre ce il dit qu'il y a trois choses, par lesquelles la nature (ne laissant rien qui ne soit signé) manifeste les hommes \& propriété de toutes choses creées, desquelles voici la première, sçavoir la chiromancie, laquelle est le vray astre \& phare de la nature, contenüe aux parties externes de l'homme comme pieds, mains, lignes, \& veines. La seconde est la physiognomie, laquelle comprend la face et le reste de la teste. La troisième \& dernière, c'est l'habitude \& proportion de tout le corps en général, laquelle dénote les mœurs, le jugement jusqu'aux plus secrettes pensées de nostre cœur, \& apres Paracelse, Ian Baptiste Aporta Napolitain, tres-celebre medecin, \& grand naturaliste en sa Physionomie, où il a travaillé au grand proffit et utilité du public. ${ }^{21}$

C'est dans la ligne de cette quête nouvelle de visibilité que s'esquissera une nouvelle conception de la «nature».

19 Bianchi 1987.

20 Astronomia Magna, trad. et cit. dans: Braun 1994, 119 et 124sq.

21 Crollius 1976,20sq. 


\section{Tableaux de mots}

Si la doctrine des signatures permet de cerner des ressemblances entre des éléments hétérogènes, leurs mises en images et en tableaux les confirment en leur donnant une évidence visuelle et graphique. Dans le cas particulier de la médication basée sur la doctrine des signatures, les liens entre la forme des plantes et les organes qu'elles peuvent traiter restent bien basés sur les similitudes; mais à partir du moment où ce système de correspondances est figé en tableaux, il tend à s'imposer comme un système ordonné, une taxinomie. Les images, lieux par excellence de la sémiologie des signatures, permettent alors à une partie des usagers de l'époque de faire l'économie d'une justification «métaphysique» de ce mode de connaissance au profit d'une attitude pratico-expérimentale. ${ }^{22}$

La distinction qui s'amorce à l'époque entre justification théorico-religieuse et utilité pratique est perceptible tout d'abord au niveau de la structure des ouvrages qui devaient servir à la formation et à la pratique quotidienne des médecins. Le Traicté des signatures de Crollius, par exemple, est structuré de manière significative en deux parties très distinctes. Le Traicté à proprement parler est une synthèse «philosophique» construite à partir essentiellement des enseignements de Paracelse. La seconde, en revanche, qui porte le titre De la signature des plantes, vise à construire un grand tableau de correspondances systématiques où se succèdent les parties du corps humain et les maladies, auxquelles correspondent les noms des plantes qui en portent la signature et qui sont donc susceptibles de les soigner, et ce dans les principales langues savantes ou ordinaires de l'époque, avec souvent l'explication de leur préparation comme médicaments. ${ }^{23}$ La manière dont Crollius structure cette seconde partie vaut la peine que l'on s'y arrête. Sur le plan du contenu, il s'agit d'une traduction pragmatique de l'exposé

22 Ceci semble être le cas en particulier dans leurs rôles pédagogique et pratique d'élaboration d'une pharmacopée systématique. Ces deux rôles étaient sans doute limités, car les ouvrages illustrés dont nous parlons étaient souvent trop onéreux ou trop rares pour servir de «manuel» à tout médecin. Mais il est acquis que les formateurs, comme Crollius par exemple, les connaissaient fort bien et s'en inspiraient largement dans la rédaction d'ouvrages graphiquement plus rudimentaires et plus rarement illustrés.

23 L'ouvrage se poursuit en empruntant en quelque sorte le chemin inverse qui part des médicaments pour arriver aux pathologies. Vient ensuite une partie consacrée aux «maladies vénéneuses, lesquelles sont souvent guéries par leur propre antidote». La partie suivante prend les allures d'une synthèse qui organise graphiquement en tableau «la correspondance du grand au petit monde, c'est-à-dire du corps humain, \& du monde». Les deux dernières explorent successivement «D'où les hommes ont pris leurs signatures», en particulier la correspondance entre leurs vertus ou caractères et celles des animaux, et enfin l'étude des correspondances entre l'homme et les animaux du point de vue de «l'usage des vomitifs \& cathartiques». 
théorique qui précède. Sur le plan de la forme, on passe d'une narration traditionnelle où l'argument est marqué en marge par des références, des résumés ou des notes, à une structure en tableau. Voici un extrait consacré aux soins des dents ${ }^{24}$ :

Les noms. Les pommes de l'acinus, ou epipetron, que les Grecs appellent àkinos, les François pomme d'Adam, représentent les dents.

Les vertus. aussi leur decoction sert et proffite de beaucoup pour les raffermir, \& oster la vilenie chancreuse, qui s'engendre autour d'icelles.

Les noms. Les noyaux du pin que les Latins appellent punus, les Grecs peùke les Italiens et Espagnols pino, les Allemands hartz baum, les Anglois pinetre, les Arabes senebar, les Flamands pinap pelboom, les Bohemiens bourouvict, ont aussi quant à eux la signature des dents.

Les vertus. \& de faict les feuilles du pin mises en decoction avec du vinaigre, font les mesmes effects que les susdites.

Les noms. La dentelee que les Latins appellent dentaria ou dentellaria, les Grecs àfullos y est aussi tres-bonne.

Les vertus. \& c'est ceste herbe à laquelle la nature a voulu donner par un admirable artifice, une racine toute garnie d'escailles.

\section{Tableaux d'images}

Pour de nombreux auteurs de l'époque comme Crollius, c'est le travail de Giovanni Battista Della Porta (1534-1615), et sa Phytognomonica en particulier, qui réalise la forme la plus achevée de la systématisation de la doctrine des signatures ${ }^{25}$. L'ouvrage présente de prime abord une forte parenté avec le travail de Crollius. On y relève pourtant des différences de taille, la plus évidente étant la présence de plus de trente xylogravures. Mais d'autres indices suggèrent que le projet graphique de Della Porta participe

24 Crollius 1976, 44sq.

25 Della Porta 1601. Outre au Phytognomonica, Crollius - qui rencontra Della Porta - fait référence au De humana physiognomia (1586). A travers ces deux ouvrages qui feront l'objet de multiples rééditions et auxquels s'ajoute encore son Magiae naturalis (1558), Della Porta sera pendant plus d'un siècle une référence incontournable pour les naturalistes. Une des raisons de cette fortune - comme de celle de Pierre Belon ou de Conrad Gesner - est d'avoir réalisé sur le plan éditorial et communicationnel l'esprit de la connaissance à laquelle aspiraient Paracelse et ses continuateurs. Cette réalisation repose pour beaucoup sur la possibilité d'actualiser les analogies sur des images concrètes. Pour donner une idée de la diffusion des travaux de Della Porta, rappelons que le De humana physiognomonia a été publié pour la première fois à Naples en 1586 puis y a été réédité en 1588, 1598, 1602, 1603,1610, 1612; ensuite à Hanovre en 1593, à Bruxelles en 1601, à Venise en 1644, à Leyde en 1645, à Rouen en 1650. Les premières traductions françaises datent de 1655 et 1665. Les complications techniques, le temps d'élaboration, la collaboration entre les métiers de la xylogravure et de l'imprimerie et les coûts engendrés par la réalisation d'ouvrages aussi richement illustrés que ceux de Della Porta font qu'ils sont encore peu nombreux à l'époque. De là sans doute découlent aussi la fascination et l'enthousiasme de Crollius, dont le Traicté des signatures n'est pas illustré. 


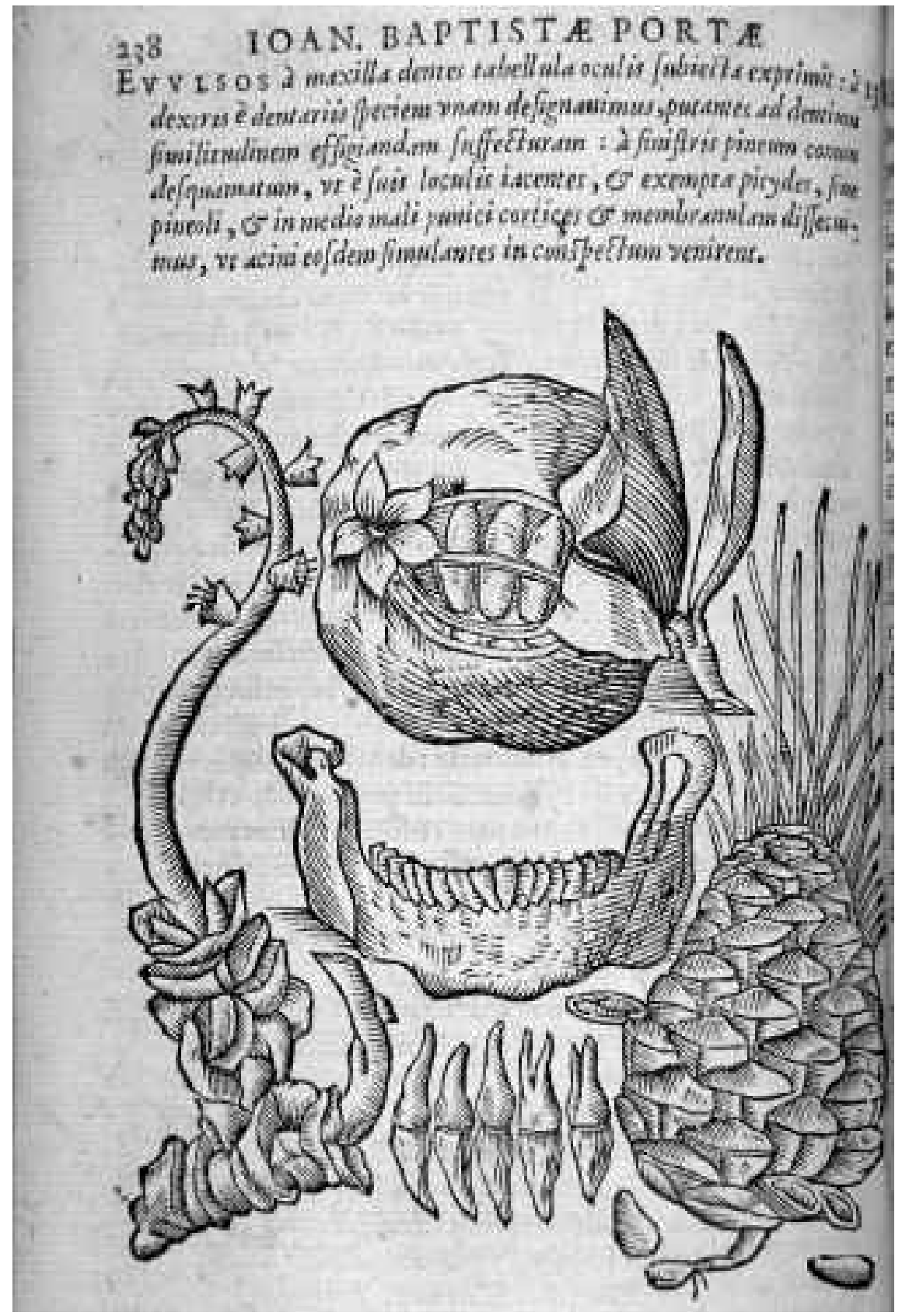

Fig. 2. Giovanni Battista Della Porta, Plantes exhibant la forme de dents, Phytognomonica (1588). 
d'une démarche un peu différente. Tout d'abord, il ne fait jamais référence à Paracelse, dont il connaît forcément les écrits et, plus surprenant, il n'utilise jamais le terme de «signature». Alors que les thèses de Paracelse et celles plus tardives de Crollius sont essentiellement basées sur une conception théologique, Della Porta n'en dit rien et privilégie la «nature» comme lieu d'observation à partir duquel fonder sa théorie ${ }^{26}$. Dans la préface du premier «livre» - le Phytognomonica en compte huit - il explique que la possibilité de remonter de l'aspect extérieur des plantes à leurs vertus thérapeutiques découle de l'observation des animaux. Certains d'entre eux, explique-t-il, savent en effet se soigner des maux ou des empoisonnements en mangeant des plantes qui paraissent bien spécifiques à leurs besoins; ils semblent donc capables de les identifier à partir de certaines caractéristiques sensibles. Cette capacité des animaux ne saurait manquer à l'homme qui doit user des mêmes sens que ses inférieurs pour reconnaître et consommer ou, au contraire, éviter des plantes. Les sens apparaissent dès lors comme les outils privilégiés de cette recherche, et c'est bien le sens de la vue que Della Porta privilégie lorsqu'il passe en revue de manière systématique toutes les plantes auxquelles, à sa connaissance, on a supposé une vertu thérapeutique basée sur un signe visible, pour ne retenir - assure-t-il son lecteur - que celles dont l'expérience aura montré l'efficacité. Le Phytognomonica est le résultat de ce processus. ${ }^{27}$

Lorsque l'on compare la gravure de Della Porta consacrée aux plantes qui permettent de soigner les dents (fig. 2) à ce que nous livre le tableau de Crollius qui lui correspond, le primat de la vue montre ses effets. La démonstration prend la forme d'une organisation de l'espace graphique qui permet au regard un parcours comparatif entre différentes plantes et différentes représentations de la dentition. La légende sert de guide:

Evulsos à maxilla dentes tabellula oculis subiecta exprimit:à dextris è dentariis speciem unam designavimus, putantes ad dentium similitudinem effigiandam suffecturam:à sinistris pineum conum desquamatum, ut è suis loculis iacentes, \& exemptae pitydes, sive pineoli, \& in medio mali punici cortices \& membranulam dissecuimus, ut acini eosdem simulantes in conspectum venirent. $^{28}$

26 Della Porta est très ancré dans son temps, au moment où la plupart des traités se proposent, comme le sien, d'inaugurer une science «neuve». Sur le sentiment de la nécessité de créer un «nouveau savoir» au XVI ${ }^{\mathrm{e}}$ siècle, voir les remarques et les références de Rossi 1996.

27 La même démarche caractérise également ses travaux de physiognomonie, à la nuance près qu'il est sans doute plus difficile à Della Porta de généraliser les liens entre un aspect du visage et le caractère d'un homme.

28 Della Porta 1601. Notons que la légende de Della Porta inverse la droite et la gauche. A moins qu'il ne s'agisse là d'une simple erreur, on peut supposer qu'il rédigeait avec les bois sous les yeux, l'image étant inversée à l'impression. 


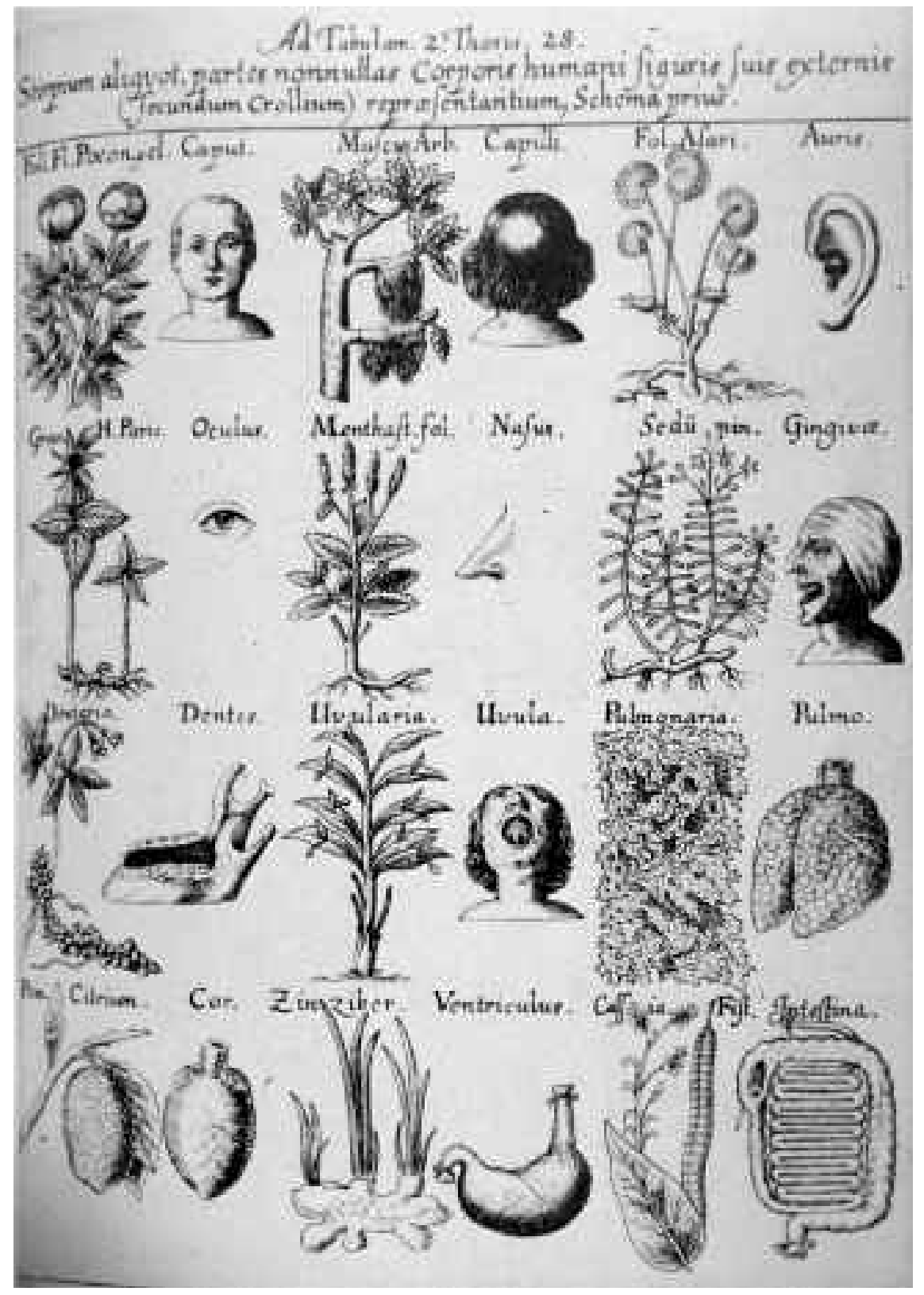

Fig. 3. W. A. Fabricius, Apophma Botanikon. De signaturis plantarum (1653). 
La dentelée, la pomme de pin et la pomme d'Adam, trois plantes mentionnées par Crollius, prennent dans la xylogravure une évidence visuelle qui rend une réelle comparaison des formes impossible - ou en tous les cas beaucoup plus difficile - pour qui ne dispose que de leur nom. Cette constatation, banale de prime abord, l'est moins lorsque l'on s'attache à la manière dont les dents sont figurées. Elles ne renvoient plus à une catégorie générique («les dents»), mais elles se déploient dans l'espace graphique en différentes versions qui relèvent d'un regard anatomique: la pomme d'Adam est comparée à une dentition alignée sur une mâchoire décharnée; la racine de la dentelée et les écailles de la pomme de pin à des dents isolées dont la racine et le col ainsi mis à nu permettent de constater la similitude avec les parties des végétaux correspondantes.

Malgré sa finesse et son ambition, tout se passe comme si la mise en image systématique de Della Porta constitue une expérience limite dans le domaine de la doctrine des signatures des plantes. En effet, mise en figures, la parenté visuelle des plantes et des organes participe d'une «rhétorique de la fidélité» qui, idéalement, doit résister à la confrontation avec les spécimens «d'après nature». Une lecture attentive de ces images nous indique pourtant que le phytognomoniste et son graveur semblent avoir été pris dans les contraintes de la ressemblance qui les ont poussés à forcer le trait. ${ }^{29}$

Cette faille de la ressemblance graphique semble apparaître dans la thèse de Wolfgang Ambrosius Fabricius en 1652 sur le thème des signatures ${ }^{30}$. Alors que Crollius s'était inspiré explicitement de Della Porta, Fabricius s'inspire du Traicté de Crollius - comme il l'indique dans la légende de la planche centrale de son travail ${ }^{13}$. Nous sommes donc pour ainsi dire placés face à une mise en images de la version de la doctrine exclusivement textuelle de Crollius. Du point de vue de la disposition générale, Fabricius reproduit la structure en tableau (fig. 3). Les couples de figures de plantes, de parties du corps et d'organes se succèdent en lignes et en colonnes. Par rapport au Phytognomonica de Della Porta, on est passé de la xylogravure à la gravure sur cuivre. Aucune des figures n'est d'ailleurs une copie de celles du

29 Sur les relations entre naturalistes et graveurs au XVI siècle, nous renvoyons au travail de Kolb qui porte surtout sur Pierre Belon, son traité richement illustré sur les oiseaux en particulier: Kolb 1996. Rappelons que le «marché des images» de l'époque est rempli de spécimens qui, à force de copies successives, finissent parfois par n'exister qu'en figure en une sorte de bibliothèque de formes archétypiques, comme ce fut le cas de la mandragore par exemple.

30 Fabricius 1997. Nous nous basons ici sur la reproduction de la planche publiée antérieurement et brièvement commentée par Bianchi 1987, pl. 2,3 et n. 112 .

31 «Stirpium aliquot, partes nonnullas corporis humani figuris suis externis (secundum Crollium) repraesentantium», Fabricius 1997. 
Napolitain. Les figures semblent reproduites à partir d'ouvrages plus récents. Il s'agit vraisemblablement pour partie de traités de botanique et d'anatomie que Fabricius avait à sa disposition, comme en témoignent les ligatures des extrémités des organes comme le «ventricule» ou les intestins ${ }^{32}$.

Il est intéressant de remarquer que, quand bien même le projet de Fabricius est, comme celui de Della Porta, d'illustrer les ressemblances morphologiques entre les plantes et les parties du corps, d'un couple de figures à l'autre, cette ressemblance est souvent ébranlée. Par exemple, si l'on peut voir quelque parenté entre la «secundinae» (le placenta) avec les «lys d'étang», la dentaria que Della Porta avait mise en regard de dents isolées (fig. 2) supporte mal la confrontation avec la mâchoire probablement recopiée d'un traité d'anatomie. Si, selon Bianchi, Fabricius semble bien être un héritier de Paracelse, son travail - comme la seconde partie de celui de Crollius apparaît comme une rationalisation de la doctrine des signatures, que toutefois, étonnamment, l'ébranlement de ces ressemblances visuelles ne semble pas remettre en question. En d'autres termes, tout se passe ici comme si la représentation graphique, qui chez Della Porta était entièrement partie prenante du «théâtre de la preuve» du fondement de la doctrine, devenait chez Fabricius un outil mnémotechnique d'un système de correspondance, comme l'était le tableau verbal de Crollius.

\section{Conclusion}

Chacun à leur manière, Della Porta, Crollius et Fabricius ont travaillé à une systématisation de la médication par les plantes, fondée sur la doctrine des signatures. Leur travail témoigne de l'exercice d'une technologie intellectuelle basée sur la construction d'un système de correspondances par l'outil du tableau. Ces tableaux, simples en apparence, sont le fruit d'une technologie graphique qui déplace la doctrine des signatures de l'ordre de la justification théorique - et théologique - vers celui de l'outil de la pratique médicale. Du point de vue de leurs usages par les praticiens, la conception analogique semble prendre les allures d'un système presque causal. Il devient en effet possible de s'y référer pratiquement de cas en cas, ou de mémoriser les rapports plantes - organes, sans pour autant qu'il soit besoin pour cela

32 Ces deux figurent tranchent de manière notoire avec la représentation des pores de la peau qui sont représentés par une version peu «anatomique» de la chair d'un écorché, dessinée en pointillé pour la cause de la figuration. Cette hétérogénéité rend plausible l'hypothèse selon laquelle ces planches sont des copies, pratique très répandue à l'époque. 
de réactiver les fondements théoriques qui ont présidé à leur élaboration ${ }^{33}$. Le tableau de correspondances transforme ainsi une conception en un instrument qui peut fonctionner comme une «boîte noire», qu'il ne s'agira d'ouvrir que lorsque le consensus sur cette conception sera mis à l'épreuve.

En d'autres termes, le phytognomoniste Della Porta comme les médecins Crollius et Fabricius entament un processus de formalisation et d'autonomisation relative de l'espace de la connaissance médicale, processus que l'histoire traditionnelle des sciences met volontiers au compte de la «révolution scientifique». Par ce bref épisode de l'histoire visuelle et scripturaire des connaissances, nous suggérons que la «modernité» scientifique et médicale s'est en fait esquissée dans une doctrine bientôt marginalisée par les nouveaux «philosophes de la nature»; sa part graphique a permis de déployer une part de la «nature» dans un nouvel espace de visibilité dont l'usage pouvait parfaitement se passer des justifications «métaphysiques» ou religieuses.

\section{Bibliographie}

Bianchi, Massimo Luigi, Signatura rerum: segni, magia e conoscenza da Paracelso a Leibniz (Roma 1987)

Braun, Lucien, Paracelse (Genève 1994)

Céard, Jean, «Analogie et zoologie chez les naturalistes de la Renaissance», dans: A. Lichnerowicz/F. Perroux/G. Gadoffre (éd.), Analogie et connaissance (Paris 1980) 75-90

Crollius, Oswald, Traicté des signatures ou vraye et vive anatomie du grand \& petit monde (Milan 1976) [or. 1609, trad. fr. 1624]

Della Porta, Giovanni Battista, Magiae naturalis (1558)

- De humana physiognomia (1586)

- Phytognomonica (Rothomagi 1601) [or. 1588]

Eisenstein, Elisabeth L., The Printing Press as an Agent of Change. Communications and Cultural Transformations in Early-Modern Europe (Cambridge 1979)

Fabricius, Wolfgang Ambrosius, Apophma Botanikon. De signaturis Plantarum (Lecce 1997) [reproduction anastatique de l'édition de Nuremberg de 1653]

Foucault, Michel, Les mots et les choses (Paris 1966)

Goody, Jack, La raison graphique (Paris 1979)

Kircher, Athanasius, Ars magna lucis et umbrae (Amstelodami ${ }^{21671)}$

Kolb, Katharina, Graveurs, artistes et hommes de science (Paris 1996)

Paracelse, Euvres complètes (Paris 1984)

Rossi, Paolo, Les philosophes et les machines (1400-1700) (Paris 1996) [or. 1962]

Shapin, Steven/Simon Schaffer, Léviathan et la pompe à air: Hobbes et Boyle entre science et politique (Paris 1993)

33 On notera la forte parenté de cette situation avec celle de la pratique médicale contemporaine où, pour prescrire un médicament, le médecin peut fort bien se passer, dans l'action, de mobiliser les théories biochimiques qui y sont cristallisées, de même que le détail de sa composition. S'il sait les maîtriser «théoriquement», pour les avoir appris, dans sa pratique quotidienne de prescription, il peut pratiquement faire l'économie de cette connaissance. 